\title{
Real and Imaginary Part of Conductivity of Strongly Interacting Bosons in Optical Lattices
}

\author{
B. Grygiel*, K. Patucha And T.A. Zaleski \\ Institute of Low Temperature and Structure Research, Polish Academy of Sciences, \\ Okólna 2, 50-422 Wrocław, Poland
}

\begin{abstract}
We calculate the conductivity of strongly interacting bosons described by the Bose-Hubbard model in a twodimensional lattice in controlled gauge potential acting as a uniform external magnetic field. In such scenario, two types of conductivity can be distinguished: intra- and inter-band. The interband contribution, usually omitted in analysis of multiband systems, appears to have a crucial role in the transport properties as its values are a few orders of magnitude greater than the intraband one.
\end{abstract}

DOI: 10.12693/APhysPolA.135.69

PACS/topics: $67.85 . \mathrm{Hj}$, 05.30.Jp, 03.75.Lm

\section{Introduction}

The cold-atom systems have been an intensively studied subject in the recent years. The development in cooling and trapping techniques $[1,2]$ has allowed for in-depth investigation of these systems. Loaded into optical lattices, they can serve as quantum simulators of the solid state systems [3], especially due to possibility of precise manipulation of their properties and lack of defects.

Introduction of artificial gauge fields by means of the Floquet engineering or photon-assisted tunneling [4, 5] has opened additional possibilities of control of behavior of particles under external potentials. Also, the measurements of the transport properties of ultracold atomic systems have been developed, leading to a successful experiments, e.g. measurements of quantized conductance in the cold-atom version of the QPC device [6] or a scanning gate microscopy method for ultracold gases [7], which can be applied to any cold-atom system.

These advances of the experimental techniques have motivated us to study the transport properties of strongly interacting bosons in optical lattice, also in the presence of the artificial magnetic field. To this end we use the Bose-Hubbard model in the quantum rotor approach, which takes into account spatial fluctuations. Thanks to this feature, we are able to properly describe the systems with multiband energy structure.

This paper is organized as follows. Section 2 contains a brief description of the quantum rotor approach to the Bose-Hubbard model and a concise derivation of the conductivity for a single- and multiband systems. Next, the dependence of the conductivity on the frequency and model parameters is presented in Sect. 3. Finally, the paper is summarized in Sect. 4.

\footnotetext{
* corresponding author
}

\section{Model and method}

We employ the Bose-Hubbard model $[8,9]$ to capture the competition between kinetic and potential energies in strongly interacting systems of bosons. The Hamiltonian of the Bose-Hubbard model is expressed in terms of creation $\hat{a}^{\dagger}$ and annihilation $\hat{a}$ operators

$$
\hat{H}=-\sum_{\langle i, j\rangle} t_{i j}\left(\hat{a}_{i}^{\dagger} \hat{a}_{j}+\text { H.c. }\right)+\frac{U}{2} \sum_{i} \hat{n}_{i}^{2}-\bar{\mu} \sum_{i} \hat{n}_{i}
$$

where $\hat{n}_{i}=\hat{a}_{i}^{\dagger} \hat{a}_{i}$ is the number operator on the $i$-th lattice site. The first term describes hopping between the sites $i$ and $j$ with hopping integral $t_{i j}$ (here, it is restricted to the nearest neighbors), the second term describes on-site repulsive interaction, and the last term contains shifted chemical potential $\bar{\mu}=\mu+U / 2$, which controls the number of particles in the system.

We use the quantum rotor approximation [10-13] to solve the Bose-Hubbard model. The method has been used to describe various physical systems e.g. high- $T_{c}$ superconductors, the Josephson junction arrays. In case of bosons in optical lattice the calculated phase diagram has been compared with the results of the quantum Monte Carlo showing good agreement between them [12]. Here, we present only a brief description of this method.

The starting point is to write the partition function in the path integral formalism with the Matsubara imaginary time [14] $0<\tau<\beta=1 / k_{\mathrm{B}} T$, where $T$ is the temperature,

$$
Z=\int[\mathcal{D} \bar{a} \mathcal{D} a] \mathrm{e}^{-S[\bar{a}, a]},
$$

where the action takes the form

$$
S[\bar{a}, a]=\int_{0}^{\beta} \mathrm{d} \tau H(\bar{a}(\tau), a(\tau))+\int_{0}^{\beta} \mathrm{d} \tau \sum_{i} \bar{a}_{i}(\tau) \frac{\partial}{\partial \tau} a_{i}(\tau) .
$$

The Hamiltonian in (3) is expressed in terms of complex fields $\bar{a}(\tau), a(\tau)$, which results from the application of the coherent state representation to the Bose-Hubbard Hamiltonian [15]. 
The next important step in the derivation is the separation of amplitude $b(\tau)$ and phase $\phi(\tau)$ degrees of freedom of the complex fields

$$
\begin{aligned}
& \bar{a}_{i}(\tau)=\mathrm{e}^{-\mathrm{i} \phi_{i}(\tau)} \bar{b}_{i}(\tau), \\
& a_{i}(\tau)=\mathrm{e}^{\mathrm{i} \phi_{i}(\tau)} b_{i}(\tau) .
\end{aligned}
$$

The amplitudes can be approximated using saddle point method $b_{i}(\tau)=b_{0}+\delta b_{i}(\tau) \approx b_{0}$. This operation is justified because the superfluid-Mott insulator transition is related to the long-range phase coherence [16]. As a result, we obtain phase dependent action of the interacting quantum rotors

$$
\begin{aligned}
& S[\phi]= \\
& \quad \int_{0}^{\beta} \mathrm{d} \tau\left[-2 J \sum_{\langle i, j\rangle} \cos \left(\phi_{j}-\phi_{i}\right)+\sum_{i}\left(\frac{\dot{\phi}_{i}^{2}}{2 U}+\frac{\mathrm{i} \bar{\mu}}{U} \dot{\phi}_{i}\right)\right],
\end{aligned}
$$

where $J=t\left|b_{0}\right|^{2}=t(t z+\bar{\mu}) / U$ is the effective coupling constant. Next, the unimodular fields $\zeta_{i}(\tau)=\mathrm{e}^{\mathrm{i} \phi_{i}(\tau)}$ can be introduced. Then, by relaxing the constraint of the unitary modulus $[17,18]$, which introduces a Lagrange multiplier $\lambda$, the equation of state $[12,19]$ and also the current-current correlation functions [13, 20-23] can be calculated.

\subsection{Multiband lattices}

The method described above can be generalized to the case of system with multiple energy bands, such as systems with gauge potentials. This is done by modifying the hopping integral by the Peierls substitution (see Sect. 2.2). As a result, the kinetic part of the modified Bose-Hubbard Hamiltonian (in the wave-vector domain) takes the following form:

$$
H_{\text {kinetic }}=\frac{t}{N_{e}} \sum_{\boldsymbol{k}}\left[a_{\boldsymbol{k}}\right]^{\dagger} \mathcal{H}(\boldsymbol{k})\left[a_{\boldsymbol{k}}\right],
$$

where $N_{e}$ denotes the number of elementary cells, $\left[a_{\boldsymbol{k}}\right]$ is the column vector of the complex fields $a_{k}^{b}$ for each site in the elementary cell $b$, and $\mathcal{H}(\boldsymbol{k})$ is the kernel of the Hamiltonian.

In the following, we supplement the Bose-Hubbard model with the Harper model [24, 25]. The Harper model describes two-dimensional, square lattice in magnetic field. The presence of the magnetic field splits the energy band into subbands, numbered by index $b$. Its strength $\alpha=p / q$, where $p, q$ are co-prime numbers, determines the number of the subbands $-q(b=1, \ldots, q)$. The energy structure of the Harper model is a fractal, known as Hofstadter butterfly.

\subsection{Conductivity}

In order to calculate the conductivity we use linear response theory

$$
\begin{aligned}
& \sigma_{x x}\left(\omega_{\nu}\right)=-\left.\frac{1}{N \beta \omega_{\nu}} \int_{0}^{\beta} \mathrm{d} \tau \mathrm{d} \tau^{\prime} \frac{\delta^{2} \ln Z}{\delta A_{x}\left(\tau^{\prime}\right) \delta A_{x}(\tau)}\right|_{\boldsymbol{A}=0} \\
& \times \mathrm{e}^{\mathrm{i} \omega_{\nu}\left(\tau-\tau^{\prime}\right)},
\end{aligned}
$$

where $\boldsymbol{A}$ denotes the vector potential of the external field. The vector potential was introduced to the BoseHubbard model through the Peierls substitution

$$
t_{i j} \rightarrow t_{i j} \exp \left(\frac{2 \pi \mathrm{i}}{\Phi_{0}} \int_{\boldsymbol{r}_{j}}^{\boldsymbol{r}_{i}} \boldsymbol{A}(\tau) \cdot \mathrm{d} \boldsymbol{l}\right),
$$

where $\Phi_{0}$ is the flux quantum. The extensive derivation of the conductivity can be found in [23]. Here, we present the final formulae. For simple lattices, without a basis, the conductivity takes the form $[16,26]$ :

$$
\begin{aligned}
& \sigma_{x x}\left(\omega_{\nu}\right)=-\frac{1}{N \beta \omega_{\nu}}\left(\frac{2 \pi J}{\Phi_{0}}\right)^{2} \sum_{\boldsymbol{k}, m}\left(\frac{\partial \varepsilon(\boldsymbol{k})}{\mathrm{d} k_{x}}\right)^{2} \\
& \times\left\{\Gamma\left(\boldsymbol{k}, \omega_{m}\right) \Gamma\left(\boldsymbol{k}, \omega_{m+\nu}\right)-\left[\Gamma\left(\boldsymbol{k}, \omega_{m}\right)\right]^{2}\right\},
\end{aligned}
$$

where the function $\Gamma^{-1}=J\left(\varepsilon(\boldsymbol{k})-\varepsilon_{0}\right)+\delta \lambda-$ $2 i v(\mu / U) \omega_{m}+\omega_{m}^{2} / U$ is a propagator calculated within the quantum rotor approach. Here, $\varepsilon(\boldsymbol{k})$ denotes the dispersion relation, $\varepsilon_{0}$ is the edge of the energy band, $\delta \lambda$ is the correction to the Lagrange multiplier (nonzero only in the Mott insulator state), and $v(\mu / U)=$ $\mu / U-\lfloor\mu / U\rfloor-1 / 2$ with $\lfloor x\rfloor$ being the floor function of $x$. After performing the summation over the Matsubara frequencies, we arrive at the formula for the regular part of the conductivity in zero-temperature limit

$\operatorname{Re} \sigma_{x x}(\omega)=\frac{2 \pi^{3} J^{2}}{\Phi_{0}^{2} U^{2}} \int \mathrm{d} E \frac{\varrho_{x x}(E)}{\Xi^{2}} \delta\left[\left(\frac{\omega}{U}\right)^{2}-4 \Xi^{2}\right]$,

$\operatorname{Im} \sigma_{x x}(\omega)=\frac{\pi^{2} J^{2}}{\Phi_{0}^{2} U^{2}} \mathcal{P} \int \mathrm{d} E \frac{\varrho_{x x}(E)}{\Xi^{3}} \frac{\frac{\omega}{U}}{\left(\frac{\omega}{U}\right)^{2}-4 \Xi^{2}}$,

where $\varrho_{x x}(E)=1 / N \sum_{\boldsymbol{k}}\left(\partial \varepsilon(\boldsymbol{k}) / \partial k_{x}\right)^{2} \delta(E-\varepsilon(\boldsymbol{k}))$ is the generalized density of states and the quantity $\Xi=\sqrt{J\left(E-\varepsilon_{0}\right) / U+\delta \lambda / U+v^{2}(\mu / U)}$ is connected to the ground-state distribution of bosons over singleparticle states. This type of conductivity results from the flow of the excitations - particle-hole pairs.

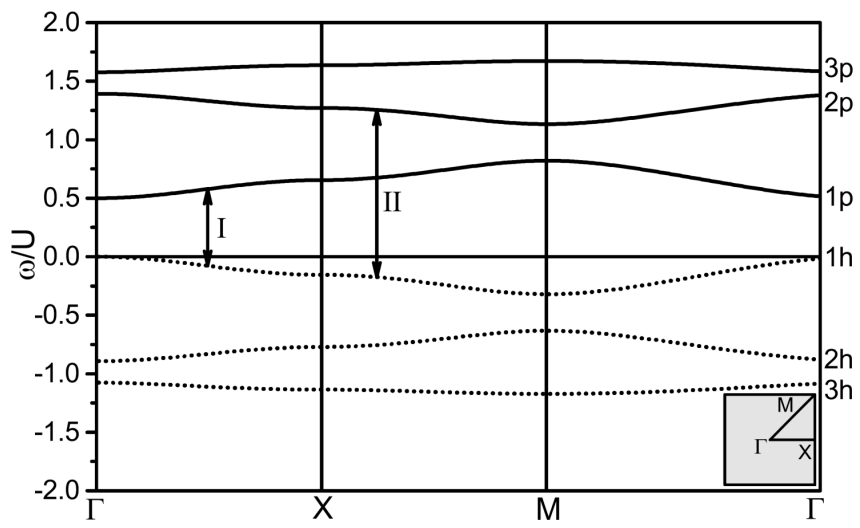

Fig. 1. The dispersion of quasiparticle and hole excitation (the spectral functions) for three-band system (Harper model, $\alpha=1 / 3$ ). Transition I - creation of an intraband particle-hole pair, II - creation of an interband pair. The inset presents the truncated Brillouin zone. 
For multiband systems (with gauge potentials, lattices with basis), an additional summation over the energy bands appears in the formula for conductivity. This leads to an additional term to the conductivity, called interband conductivity, which is related to the excitations of particle-hole pairs between different bands (see Fig. 1). It takes the following form:

$$
\begin{aligned}
& \sigma_{x x}^{\text {inter }}\left(\omega_{\nu}\right)=-\frac{1}{N \beta \omega_{\nu}}\left(\frac{2 \pi J}{\Phi_{0}}\right)^{2} \sum_{\boldsymbol{k}, m} \sum_{b \neq b^{\prime}} M_{x x}^{b b^{\prime}}(\boldsymbol{k}) \\
& \quad \times\left[\Gamma_{b}\left(\boldsymbol{k}, \omega_{m}\right) \Gamma_{b^{\prime}}\left(\boldsymbol{k}, \omega_{m+\nu}\right)-\Gamma_{b}\left(\boldsymbol{k}, \omega_{m}\right) \Gamma_{b^{\prime}}\left(\boldsymbol{k}, \omega_{m}\right)\right],
\end{aligned}
$$

where the summation $\sum_{b \neq b^{\prime}}$ runs over pairs of bands. The quantity $M_{x x}^{b b^{\prime}}(\boldsymbol{k})=\left|\left\langle b\left|\left(\partial \mathcal{H}(\boldsymbol{k}) / \partial k_{x}\right)\right| b^{\prime}\right\rangle\right|^{2}$ is the analogue of the weight present in the generalized density of states. Performing the summation over the Matsubara frequencies yields the formulae for the real and imaginary parts of interband conductivity for $T=0$ :

$$
\begin{aligned}
& \operatorname{Re} \sigma_{x x}^{\text {inter }}(\omega)=\frac{2 \pi^{3} J^{2}}{\Phi_{0}^{2} U^{2}} \frac{1}{N} \sum_{\boldsymbol{k}} \sum_{b \neq b^{\prime}} \frac{M_{x x}^{b b^{\prime}}(\boldsymbol{k})}{\Xi_{b} \Xi_{b^{\prime}}} \\
& \times \delta\left[\left(\frac{\omega}{U}\right)^{2}-\left(\Xi_{b}+\Xi_{b^{\prime}}\right)^{2}\right], \\
& \operatorname{Im} \sigma_{x x}^{\text {inter }}(\omega)=\frac{2 \pi^{2} J^{2}}{\Phi_{0}^{2} U^{2}} \frac{1}{N} \sum_{k} \sum_{b \neq b^{\prime}} \frac{M_{x x}^{b b^{\prime}}(\boldsymbol{k})}{\Xi_{b} \Xi_{b^{\prime}}\left(\Xi_{b}+\Xi_{b^{\prime}}\right)} \\
& \quad \times \frac{\frac{\omega}{U}}{\left(\frac{\omega}{U}\right)^{2}-\left(\Xi_{b}+\Xi_{b^{\prime}}\right)^{2}} .
\end{aligned}
$$

\section{Results}

This section contains a brief analysis of the behavior of the conductivity as a function of the frequency and the parameters of the Bose-Hubbard model. Figures 2 and 3 present the dependence of the real and imaginary parts of the conductivity for a square lattice in the superfluid and Mott insulator phases, respectively.

The real part of the conductivity is related to the current response, which is in phase with the external probe field. It exhibits a lobe-like transport channels with the characteristic energy gap for the particlehole excitation $\omega_{g} / U=2 \sqrt{v^{2}(\mu / U)+\delta \lambda / U}$. This gap is greater in the Mott insulator phase. Also, in the Mott insulator phase the values of the conductivity are smaller. The imaginary part, related to the current in anti-phase with the external field, exhibits a change in the convexity in the middle of the lobe of the real part.

The behavior of the conductivity for multiband systems is presented for the Harper model for magnetic field $\alpha=1 / 3$ (in the superfluid phase). In Fig. 4 the behavior of the intraband part is shown. Three transport channels in the real part of $\sigma_{x x}$ can be observed. Each one is connected to the generation of a particlehole pair, e.g. the first lobe results from the generation of the particle in the first particle-band and the hole in

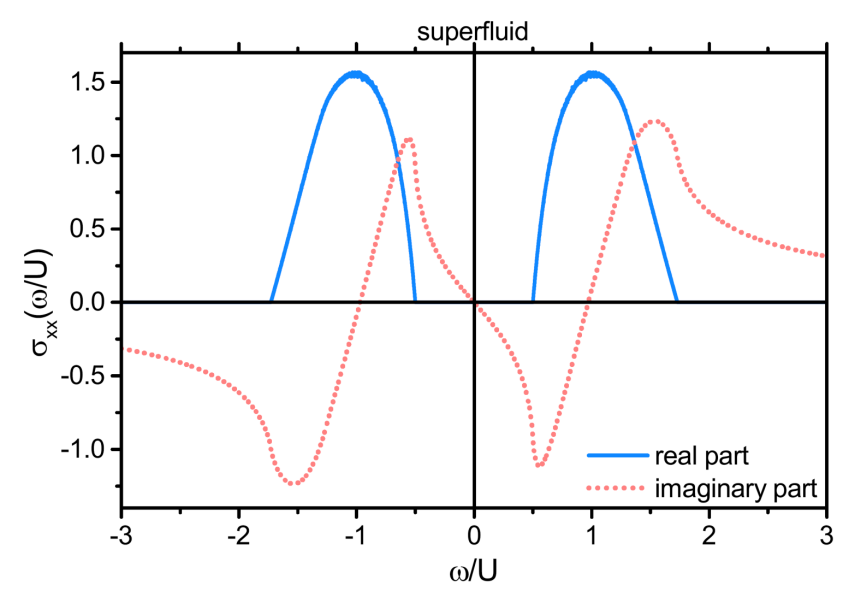

Fig. 2. The behavior of the regular part of the conductivity for two-dimensional square lattice for $T=0$ in the superfluid phase. The conductivity is expressed in the units of $\left[1 / \Phi_{0}^{2}\right]$.

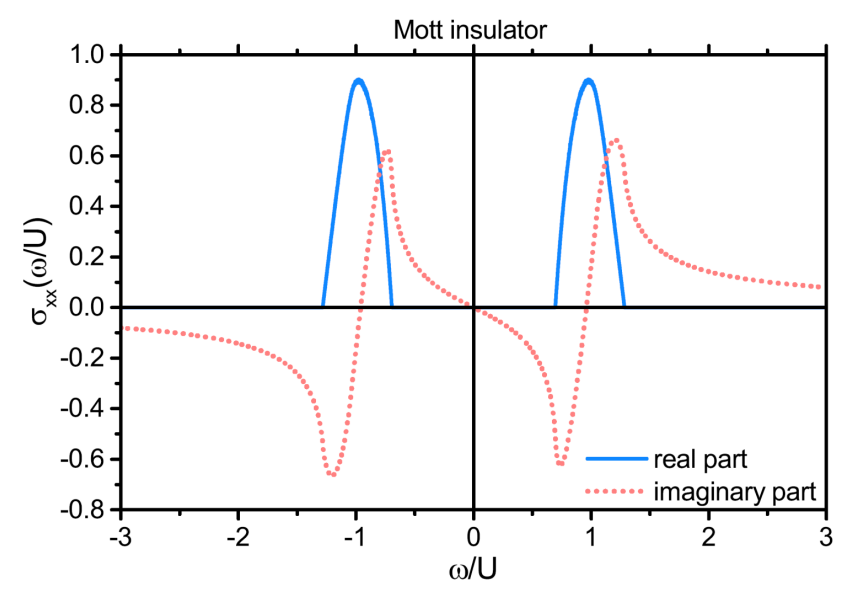

Fig. 3. As in Fig. 2, but for the Mott insulator phase.

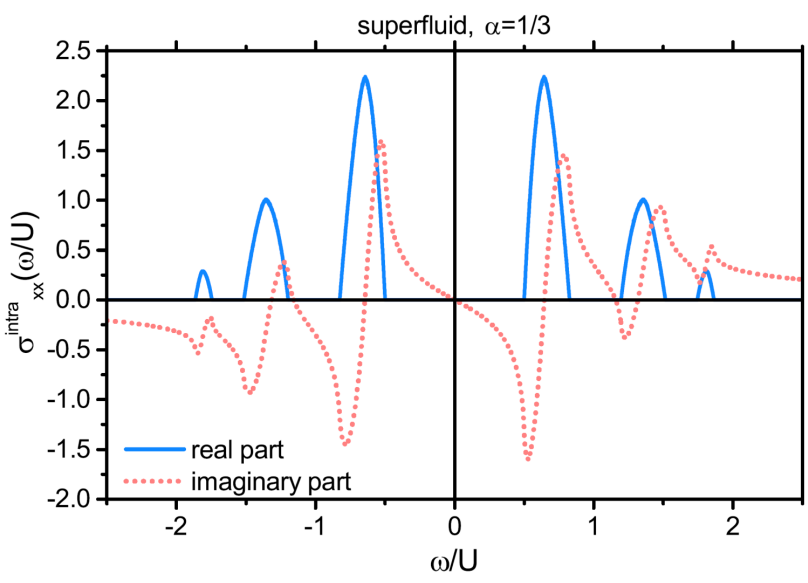

Fig. 4. The behavior of the regular, intraband part of the conductivity for two-dimensional square lattice in magnetic field $\alpha=1 / 3$ for $T=0$ in the superfluid phase. The conductivity is expressed in the units of $\left[1 / \Phi_{0}^{2}\right]$. 


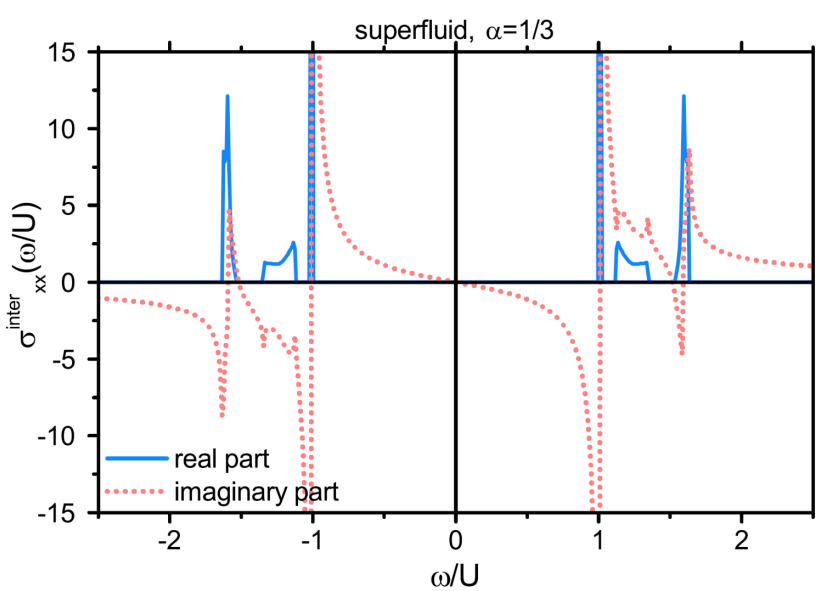

Fig. 5. As in Fig. 4, but for the interband part of the conductivity.

the first hole-band (see transition I in Fig. 1). In the dependence of the imaginary part, there can be observed a "response" related to the consecutive transport channels in the real part.

In Fig. 5 the dependence of the interband part of the conductivity on the frequency of the external field is presented. In the real part, three transport channels are present. Each one lies between the respective pair of the intraband channels. Here, the generation of the particlehole pair between different bands is responsible for the shape of the channels. For example, the transition II in Fig. 1 corresponds to the first transport channel in Fig. 5. Especially, the similar curvature of the first holeand the second particle-band explains why the first interband channel is narrow and high. The imaginary part of the interband conductivity exhibits cusps, which correspond to the sharp changes of the real part. This can be understood through the Kramers-Krönig relations. The values of the interband conductivity are at least one order of magnitude greater compared with the intraband conductivty.

\section{Summary}

The conductivity of strongly interacting bosons in optical lattice has been studied using the Bose-Hubbard model in the quantum rotor approach. The influence of the multiband energy structure of the Harper model has been taken into consideration by combining the Harper model with the Bose-Hubbard Hamiltonian.

By means of the linear response theory the conductivity of the system was calculated. Thanks to the quantum rotor approximation, the conductivity has been determined both in the superfluid and in the Mott insulator phases. For the Harper-Bose-Hubbard model the conductivity consists of two types of terms, intraand interband, where the latter is not present for a simple square lattice. The interband part is heavily influenced by the relative quasiparticle- and hole- band curvature. The transport properties of the multiband systems are dominated by the interband part, which can be a few orders of magnitude higher than the intraband part.

Recently, a number of experimental methods has been developed for measurements of transport phenomena with high spatial resolution. However, in order to verify the results of the present paper, a different approach is required that allows for determination of conductivity as a function of frequency. Such a method has been proposed using a phase modulation of the optical lattice to investigate current self-correlations [27]. This, in principle, could allow for visualization of various transport channels including intra and interband contributions.

\section{References}

[1] C. Pethick, H. Smith, Bose-Einstein Condensation in Dilute Gases, Cambridge University Press, 2002.

[2] I. Bloch, J. Dalibard, W. Zwerger, Rev. Mod. Phys. 80, 885 (2008).

[3] M. Lewenstein, A. Sanpera, V. Ahufinger, B. Damski, A. Sen(De), U. Sen, Adv. Phys. 56, 243 (2007).

[4] G. Jotzu, M. Messer, R. Desbuquois, M. Lebrat, T. Uehlinger, D. Greif, T. Esslinger, Nature 515, 237 (2014).

[5] M. Aidelsburger, M. Atala, M. Lohse, J.T. Barreiro, B. Paredes, I. Bloch, Phys. Rev. Lett. 111, 185301 (2013).

[6] S. Krinner, D. Stadler, D. Husmann, J.-P. Brantut, T. Esslinger, Nature 517, 64 (2015).

[7] S. Häusler, S. Nakajima, M. Lebrat, D. Husmann, S. Krinner, T. Esslinger, J.-P. Brantut, Phys. Rev. Lett. 119, 030403 (2017).

[8] M.P.A. Fisher, P.B. Weichman, G. Grinstein, D.S. Fisher, Phys. Rev. B 40, 546 (1989).

[9] D. Jaksch, C. Bruder, J.I. Cirac, C.W. Gardiner, P. Zoller, Phys. Rev. Lett. 81, 3108 (1998).

[10] T.K. Kopeć, J.V. José, Phys. Rev. B 60, 7473 (1999).

[11] T.K. Kopeć, Phys. Rev. B 70, 054518 (2004).

[12] T.P. Polak, T.K. Kopeć, Phys. Rev. B 76, 094503 (2007).

[13] T.A. Zaleski, T.K. Kopeć, Phys. Rev. A 84, 053613 (2011).

[14] A.L. Fetter, J.D. Walecka, Quantum Theory of ManyParticle Systems, McGraw-Hill, San Francisco 1971.

[15] H. Kleinert, Path Integrals in Quantum Mechanics, Statistics, Polymer Physics, and Financial Markets, World Sci., Singapore 2006.

[16] A.P. Kampf, G.T. Zimanyi, Phys. Rev. B 47, 279 (1993).

[17] T. Vojta, Phys. Rev. B 53, 710 (1996).

[18] T.M. Nieuwenhuizen, Phys. Rev. Lett. 74, 4293 (1995).

[19] B. Grygiel, K. Patucha, T.A. Zaleski, Phys. Rev. A 93, 053607 (2016).

[20] T.A. Zaleski, J. Phys. B At. Mol. Opt. Phys. 45, 145303 (2012). 
[21] T.P. Polak, T.A. Zaleski, Phys. Rev. A 87, 033614 (2013).

[22] T.A. Zaleski, T.K. Kopeć, Physica B 433, 37 (2014).

[23] B. Grygiel, K. Patucha, T.A. Zaleski, Phys. Rev. B 96, 094520 (2017).

[24] P.G. Harper, Proc. Phys. Soc. A 68, 874 (1955).
[25] D.R. Hofstadter, Phys. Rev. B 14, 2239 (1976).

[26] A.S. Sajna, T.P. Polak, R. Micnas, Phys. Rev. A 89, 023631 (2014).

[27] A. Tokuno, T. Giamarchi, Phys. Rev. Lett. 106, 205301 (2011). 